\title{
Improving Adhesive Properties of Cellulose Nanocrystals Modifying by 3-Glycidoxypropyltrimethoxy Silane (KH-560) Coupling Agents
}

\author{
Tariq Aziz ${ }^{*}$, Mudassir Iqbal ${ }^{1}$, Roh Ullah², Muhammad Imran Jamil ${ }^{1}$, Muhammad Raheel ${ }^{3}$, \\ Amjad Ali ${ }^{4}$, Farman Ullah Khan ${ }^{5}$, Asmat Ullah ${ }^{6}$ and Nasrullah Wazir ${ }^{7}$ \\ ${ }^{1}$ College of Chemical and Biological Engineering Zhejiang University, China
}

${ }^{2}$ School of Chemical and Biological Engineering, Beijing Institute of Technology (BIT), China

${ }^{3}$ Baluchistan University of Information Technology, Engineering and Management Sciences (BUITEMS), Pakistan

${ }^{4}$ School of Material Science \& Engineering, Jiangsu University, China

${ }^{5}$ Department of Chemistry, University of Science and Technology Bannu 28000, Pakistan

${ }^{6}$ School of Pharmacy. Xi'an Jiaotong University Shaanxi, China

${ }^{7}$ School of Physics, Beijing Institute of Technology, China

*Corresponding author: Tariq Aziz, College of Chemical and Biological Engineering Zhejiang University, Hangzhou, 310027, China

\section{ARTICLE INFO}

Received: 幽 February 11, 2021

Published: 慧 February 18, 2021

Citation: Tariq Aziz, Mudassir Iqbal, Roh Ullah, Muhammad Imran Jamil, Muhammad Raheel, et al. Improving Adhesive Properties of Cellulose Nanocrystals Modifying by 3-Glycidoxypropyltrimethoxy Silane (KH-560) Coupling Agents. Biomed J Sci \& Tech Res 34(1)-2021. BJSTR. MS.ID.005492.

Keywords: Adhesive Properties; Cellulose Nanocrystals; Industrial; KH-560 Coupling Agent; Modification

\begin{abstract}
Cellulose nanocrystals (CNCs) is one of the outstanding nanomaterial. So far, much researcher nowadays has been developed their properties. These properties make CNCs a promising resource to replace fossil resources for the production of industrial materials and chemicals. Here, we firstly modified cellulose nanocrystals with 3-Glycidoxypropyltrimethoxy (KH-560) silane coupling agent. Chemical composition and surface morphologies of cellulose nanocrystals were analyzed and characterized by FT-IR, and SEM. The result indicated that polar hydroxyl groups were successfully introduced on the cellulose nanocrystals surface. The SEM results confirmed silane coupling agent was successfully grafted on cellulose nanocrystals. Modified CNCs demonstrated an excellent tensile strength and modulus exhibited by $1 \mathrm{wt} \%$ modified cellulose nanocrystals composites. This novel procedure improves the dispersion of cellulose nanocrystals by modification and at the same time enhance the interfacial adhesion. Result shows increases more over than the native cellulose nanocrystals by modifying CNCs with $\mathrm{KH}-$ 560 coupling agent. This study lays a foundation towards full polymers fabrication and consumptions most desirable in adhesive and mechanical industrial fields.
\end{abstract}

\section{Introduction}

Cellulose nanocrystals (CNCs) obtained from natural bio resources building blocks for the design of new biomaterials in nanotechnology [1]. Their widths varying from 5 to $20 \mathrm{~nm}$ and lengths from $100 \mathrm{~nm}$ to $1-2 \mu \mathrm{m}$ [2]. Cellulose nanocrystals used for several years both in modified and unmodified form for the development and products which is useful for our society [3,4]. CNCs extracted from abundant cellulose nanofillers that is sustainable, biodegradable and has excellent strength-to-weight ratio used as reinforcing for epoxy composites [5]. Recently it has been shown that cellulose nanocrystals a sustainable bio sourced material, 
could serve as a valuable colloidal stabilizer [6,7]. It attracted a dynamic attention in developing lightweight and high performance nano composite due to their excellent mechanical performance, biodegradable and renewable abilities [8,9]. Cellulose nanocrystals materials are widely used due to their unusual properties, such as high specific surface area, static mechanical properties, fatigue resistance and permeability $[10,11]$. In several studies' CNCs have been incorporated into polymer matrices as a reinforcing agent, due to hydrophilic characterization low or higher molecular weight molecules has been attempted to achieve good dispersion and adhesion in the non-polar matrices [12,13]. Cellulose nanocrystals is favorable reinforcing material for production of bio-based composite material because of its high strength and stiffness $[14,15]$.

Also, cellulose nanocrystals are bio sourced safe nanomaterials of great research interest in both academic and industrial sectors. Their unique properties and future commercialization prospects have recently led potentially industrial production $[16,17]$. Nowadays epoxy resin are largely used in our daily life, like adhesives, adhesion between different matrix, excellent performance in mechanical properties $[18,19]$. It is used for making advanced types of composites such as coating in electric applications because of the versatility in curing, high excellent modulus, low shrinkage and high tunable $[20,21]$. Epoxy resin, most commonly thermosetting materials especially in the fiber manufacturing materials due to their excellent thermal and chemical resistance $[22,23]$. However, relatively high content of hydroxyl group after cured, epoxy resin perform high surface energy which compel their application in some areas such as anti-fouling coating [24]. Among organic coating, epoxy resin are used on large quantity in protective coating due to their excellent properties like ease of cure, high temperature performance [25,26]. Epoxy resin system is also used in high voltages transformers, power apparatus and cable terminations. Surface modification of fillers or crystals having coreshell structures in polymers can greatly enhance the performances such as electrical and thermal conductivity. Much efforts has been carried out to develop new insulating materials and related fabrication method for enhancing performance $[27,28]$.

Epoxy resin can be used in preparation of organic-inorganic hybrids with potential applications in coating and composite industries because of their excellent mechanical properties [29]. It is the best choice used for anti-corrosion and corrosion resistance. They become a favored choice for long term, cost effective in various industrial applications [30,31]. The purpose of this study is to prepare new modified cellulose nanocrystals CNCs with 3-Glycidoxypropyltrimethoxy silane (KH-560) coupling agent for improving adhesive properties. Because both cellulose nanocrystals CNCs and coupling agents have significant applications is expected to provide a combination of high stiffness and strength to the epoxy resin which is most desirable in many industrial levels and giving excellent result in such field. The present study of cellulose nanocrystals CNCs is modified with silane (KH-560) coupling agent and reacted with E-51 epoxy resin analyzing their adhesive properties with the help of different characterization. Epoxy resin is a polymeric material with extensive applications was choosing as the model polymer matrix. Modified cellulose nanocrystals CNCs is expected to provide a combination of high stiffness and strength to the epoxy resin which is most desirable in many industrial applications.

\section{Materials and Methods}

Cellulose nanocrystals (CNCs) were obtained from Science $\mathrm{K}$ Co. Ltd. Wzhangzhen China. There average length is $10-250 \mathrm{~nm}$ and average diameter with $7.5 \mathrm{~nm}$. CNCs was firstly dried at $80^{\circ} \mathrm{C}$ for $8 \mathrm{~h}$ in vacuumed oven to remove the impurities and moisture. E-51 epoxy resin from sigma Aldrich. 3-Glycidoxypropyltrimethoxysilane (KH560) from Aladdin Industrial Corporation Shanghai's. Analytical pure reagents including ethanol, acetic acid was obtained from the Aladdin reagent (Shanghai, China). All chemicals were used as received. Lab made distilled water were used.

\section{Modification of Cellulose Nanocrystals With (KH-560)}

Modified cellulose nanocrystal (MCNCs) was prepared in a single-necked $150 \mathrm{ml}$ round bottom flask. Firstly taking $100 \mathrm{ml}$ distilled water with $5 \mathrm{ml}$ 3-Glycidoxypropyltrimethoxy silane $(\mathrm{KH}-$ 560 ) putting some drops of acetic acid to maintain the $\mathrm{pH} 4$ stirring for one h. After that neat dried CNCs were immersed in a singlenecked round bottom flask and placed in sonication for 20-30 min, so that cellulose nanocrystals were fully disperse in the solution. Then stirred for the next $150 \mathrm{~min}$ at natural temperature. After that, the product was put into the centrifugation sample for washing two times by ethanol absolute ( $96 \%$ purity) and one time with distilled water. One round was $10 \mathrm{~min}$ at $5000 \mathrm{rpm}$ in centrifugation (Shanghai Anting scientific instrument factory) to remove any excess of silane from the cellulose nanocrystals. After washing the product in the centrifugation put it into hot air oven for 4-5 h to remove the impurities, maintains the temperature at $100^{\circ} \mathrm{C}$. The powder again put into the vacuumed oven with $80^{\circ} \mathrm{C}$ for the next 4-6 $\mathrm{h}$ to remove the remaining impurities and then subsequently preserved in sample glass bottle from moisture for further use.

\section{Samples Preparation}

A simulating industrial setup for steel plates was designed from Hangzhou iron and steel group Co. Ltd. The steel plates were polished and was dried at room temperature and subsequently preserved in plastic bags from moisture for further use. According to test methods for adhesive properties of MCNCs embedded with coated steel plates was polish molded into 3 standard testing. E-51 (DGEBA) epoxy resin $1 \mathrm{~g}$ and MCNCs with $1 \%, 3 \%$ and $5 \%$ was taken in the sample glass bottle and sonication at natural temperature for $1 \mathrm{~h}$ and then stirred at room temperature for the next $2 \mathrm{~h}$. After that, $0.2 \mathrm{~g}$ of triethylenetetramine (TETA) added drop by drop and slowly stirred for the next 10-15 min for complete curing. Then 
place it in vacuumed oven for 15-20 min maintain the temperature up-to $45^{\circ} \mathrm{C}$ to remove the air bubbles from the solution, after that the complete mixture was poured by glass rod on steel plates with the length of $1.5 \mathrm{~cm}$ and width $2.3 \mathrm{~cm}$ into three different molds for curing. Curing process was carried out in an oven at $120^{\circ} \mathrm{C}$ for $1 \mathrm{~h}$. After that, the samples were cooled down naturally to room temperature. These standard testing steel plates were placed at room temperature for 3-4 days before testing.

\section{E-51 (DGEBA) Epoxy Resin Used in This Work (Scheme}

1)

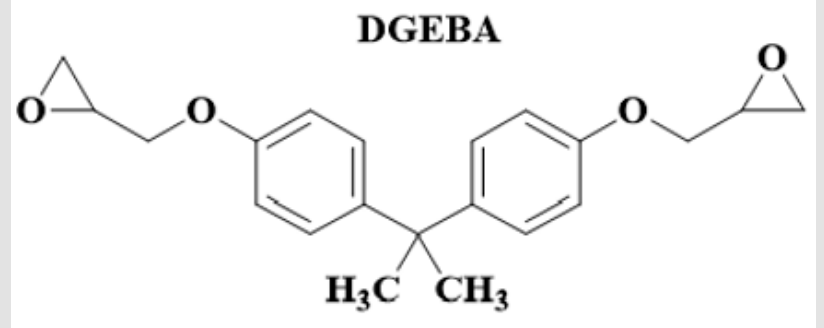

Scheme 1: E-51 (DGEBA) epoxy resin used in this work.

\section{Characterizations}

The structure of E-51 epoxy resin, both silane coupling agent (KH-560) were analyzed by the Fourier transform infrared spectroscopy (FT-IR). The infrared spectra recorded on a Nicolet-5700 FT-IR spectrophotometer by the KBr-pellet method and scanned from $4000-500 \mathrm{~cm}^{-1}$ with a resolution superior to $0.5 \mathrm{~cm}^{-1}$. The SEM images were obtained on scanning electron microscope (SEM, SU-3500) with X-ray spectrometer, coating of gold surface on the specimen were applied. The morphologies of the fracture surface images of silane coupling agent (KH-560) sized were obtained on this SEM. For adhesive properties, universal material testing machine (Zwick/roell Z020) were used for such properties like tensile strength, modulus and impact strength were analyzed.

\section{Result and Discussion}

\section{Modification of Cellulose Nanocrystals With KH-560 Coupling Agent}

The different functional groups present in neat cellulose nanocrystals (NCNCs) and MCNCs were studied by FTIR. The typical
FTIR spectra of NCNCs and MCNCs is shown in Figure 1. Neat CNCs showed a strong peak appeared at $1750 \mathrm{~cm}^{-1}$ was ascribed to the $\mathrm{C}=\mathrm{C}$ group stretching vibration of the ester and carboxylic group present in MCNCs [32,33]. The appearance of this peak confirmed the successful synthesis of the MCNCs.

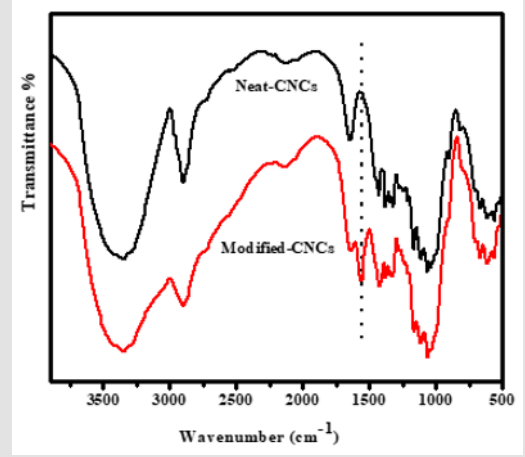

Figure 1: FT-IR of neat and modified cellulose nanocrystals.

\section{Scanning Electron Microscope (SEM)}

The Scanning electron microscope (SEM) images were obtained on field emission scanning electron microscope (SU3500) for neat cellulose nanocrystals. After sputter coating of gold on the specimen's surface. The morphologies of the surface images of neat cellulose nanocrystals were obtained and clearly showing the surfaces of cellulose nanocrystals. The morphologies of the surface of neat and modified cellulose nanocrystals images were obtained and clearly showed the surfaces morphologies, reacted with aforementioned epoxies. The neat CNCs surface is much rougher than the modified CNCs. In general, their combination has a favorable effect on the performance of composites, which results an increase in the adhesive properties as shown in Figure 2 [34]. SEM of cellulose nanocrystals matrix appeared with epoxy resin, but their diameter decreased after spreading to varying degrees in the epoxy. However, as additional CNCs aggregation occurred, which prevented the formation of a homogeneous mixture. In general, their combination has a favorable effect on the performance of composites. However, better dispersion was obtained using a modified epoxy resin with low CNCs content. Low concentrations of CNCs are more appropriate than higher and aggregation resulted with increasing concentrations. The presence of multiple break lines in the epoxy surface indicates the hardness of the material. The modified epoxy resin particles exhibited bumps and collapsed morphology.

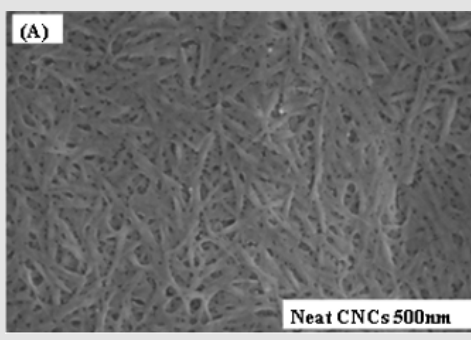

Figure 2: (A) Neat CNCs (B) Modified CNCs.

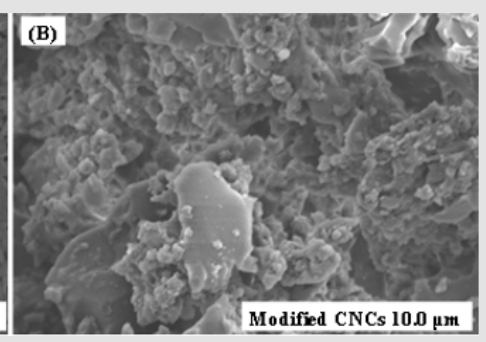




\section{Adhesive Properties of Modified Cellulose Nanocrystals}

Here, we have also evaluated the adhesives strength of neat and modified CNCs, because they have an assured tensile modulus, strength and elongation at break. The average adhesive strength is mentioned in Figure-03. (A-B), using KH-560 silane coupling agent with the CNCs content, while triethylenetetramine (TETA) used as a curing agent. The epoxy resins containing neat and modified CNCs at 1, 3 and 5 weight percent (wt \%) to evaluate the effect of enhancement potential. With the introduction of tensile modulus and strength at maximum load using steel plates. Modified CNCs has an obvious enhancement effect with 1 wt $\%$ and 3 wt $\%$ and 5 $\mathrm{wt} \%$ on tensile modulus, but for tensile strength after adding 3\% started agglomeration in the epoxy medium $[35,36]$. The maximum value observed for nanocomposites reinforced with all the samples [37]. The tensile data shows that all the modified CNCs samples had a higher tensile strength than the standard one. The results of modified CNCs binding themselves through $-\mathrm{OH}$, creating a high strength linkage, with potentially interaction among them, increasing the adhesive properties. The increasing of modified CNCs loading result in agglomeration between particles, which produce weakness in the material, explaining the comparative decrease in adhesive with individual loading.
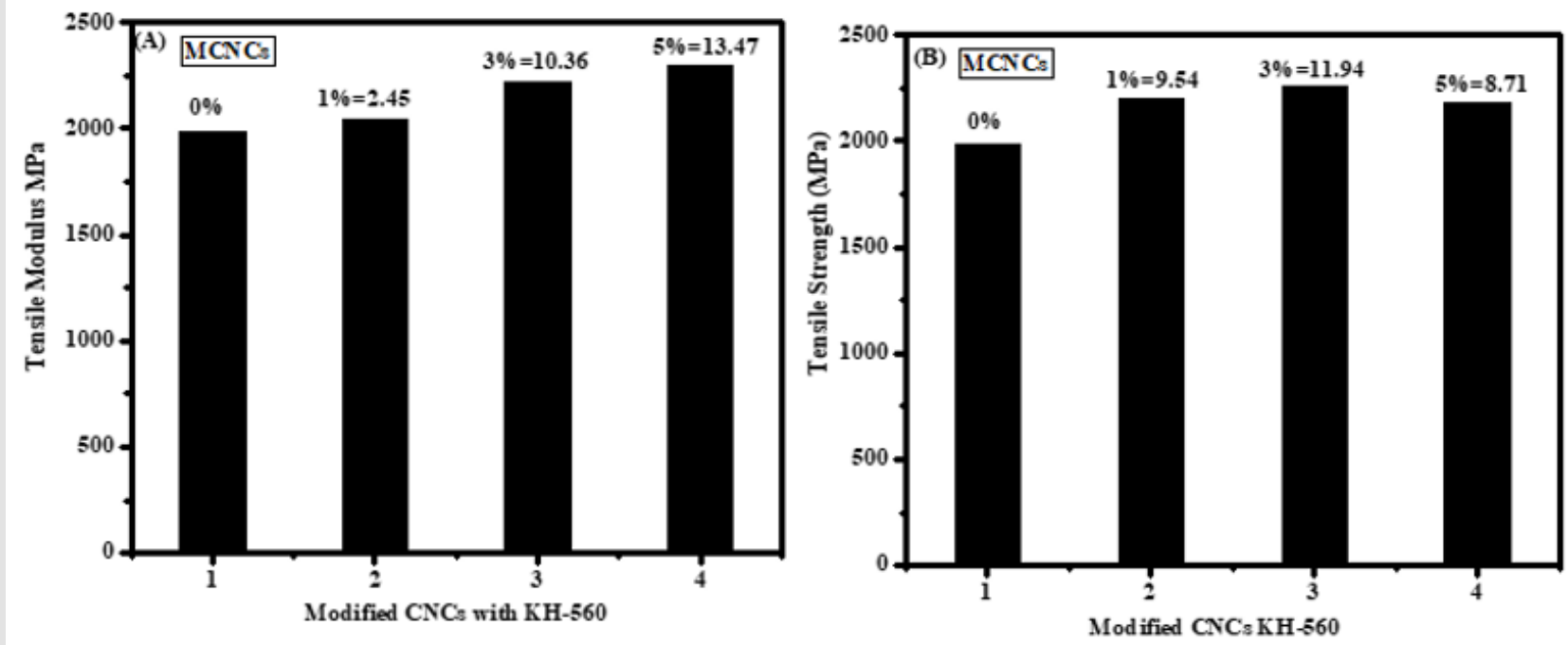

Figure 3: (A), Tensile modulus (B) Tensile Strength.

\section{Elongation at Break}

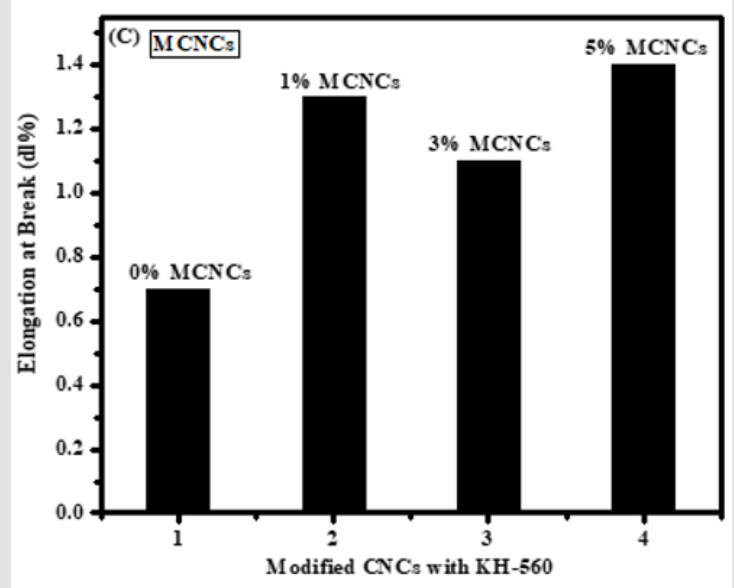

Figure 3: (C), Modified cellulose nanocrystals Elongation at break.

The elongation break corresponds to the percentage of expansion of the initial span of the modified CNCs samples [38,39].
The duration of the interval corresponds to the percentage growth of the modified cellulose nanocrystals. In Figure 3. (C), all samples sizes improved permanently compared to the standard sample. A moderate upward trend was observed, which improved the dispersion of the modified CNCs in the epoxy system. Responsible for the differences in the inherent characteristics of the modified CNCs. Cellulose nanocrystals are generally considered strong, hard and fragile. The distribution of modified CNCs is good, resulting in nanomaterials of different length [40-42].

\section{Conclusion}

Surface morphology of cellulose nanocrystals obtained from industrial cotton have observed large mesh formations in transmission electron microscopy. The excess amount of CNCs presented aggregation behavior having highly rough surface, however, good dispersion observed in low concentrations of CNCs in DGEBA epoxy resins. The corresponding coupling agent bears a long chain structure of benzene ring in molecular structure, granting compatibility to CNCs with epoxy, contributing to the dispersion state in the matrix. In addition, higher loading of modified CNCs have increased the surface roughness, irrespective 
of the surface modification method. The surface modification has been confirmed through different techniques. Modified CNCs have obvious enhancement effect with $1 \mathrm{wt} \%$ and $3 \mathrm{wt} \%$ on tensile strength, but addition of $5 \mathrm{wt} \%$ caused agglomeration in the epoxy medium. The effects of KH-560 on the adhesive and mechanical properties with epoxy systems indicated effectively, improve the toughness of the epoxy system.

\section{Statement}

We have no conflict of interest.

\section{Acknowledgments}

This research was funded by the State Key Laboratory of Chemical Engineering, Zhejiang University 310027 Hangzhou, China

\section{Sources of Financial Funding and Support}

This research work is not funded by any agency.

\section{References}

1. Abraham E, Kam D, Nevo Y, Slattegard R, Rivkin A, et al. (2016) Highly modified cellulose nanocrystals and formation of epoxy nanocrystalline cellulose (CNC) nanocomposites. ACS Appl Mater Inter 8 (41): 2808628095.

2. Du WB, Guo J, Li HM, Gao Y (2017) Heterogeneously modified cellulose nanocrystals-stabilized pickering emulsion: preparation and their template application for the creation of PS microspheres with aminorich surfaces. ACS Sustain Chem Eng 5 (9): 7514-7523.

3. Sunasee R, Hemraz UD (2018) Synthetic strategies for the fabrication of cationic surface-modified cellulose nanocrystals. Fibers 6 (1): 1-12.

4. Aziz T, Ullah A, Fan H, Ullah R, Haq F, et al. (2021) Cellulose nanocrystals applications in health, medicine and catalysis. J Polym Environ 1-10.

5. Yue L, Maiorana A, Khelifa F, Patel A, Raquez JM, et al. (2018) Surfacemodified cellulose nanocrystals for biobased epoxy nanocomposites. Polymer 134: 155-162.

6. Zhang Z, Tam KC, Wang XS, Sebe G (2018) Inverse pickering emulsions stabilized by cinnamate modified cellulose nanocrystals as templates to prepare silica colloidosomes. ACS Sustain Chem Eng 6 (2): 2583-2590.

7. Zheng J, Zhang X, Cao J, Chen R, Aziz T, et al. (2021) Behavior of epoxy resin filled with nano-SiO2 treated with a eugenol epoxy silane. J Appl Polym Sci 138 (14): 50138

8. Zhou L, He H, Li MC, Huang SW, Mei CT, et al. (2018) Enhancing mechanical properties of poly(lactic acid) through its in-situ crosslinking with maleic anhydride-modified cellulose nanocrystals from cottonseed hulls. Ind Crop Prod 112: 449-459.

9. Aziz T, Fan H, Khan FU, Haroon M, Cheng L, et al. (2019) Modified silicone oil types, mechanical properties and applications. Polymer Bulletin 76 (4): 2129-2145.

10. Abraham E, Weber DE, Sharon S, Lapidot S, Shoseyov O, et al. (2017) Multifunctional cellulosic scaffolds from modified cellulose nanocrystals. ACS Appl Mater Inter 9 (3): 2010-2015.

11. Aziz T, Fan H, Haq F, Khan FU, Numan A, et al. (2020) Adhesive properties of poly (methyl silsesquioxanes)/bio-based epoxy nanocomposites. Iranian Polymer Journal 29 (10): 911-918.

12. Boujemaoui A, Sanchez CC, Engstrom J, Bruce C, Fogelstrom L, et al. (2017) Polycaprolactone nanocomposites reinforced with cellulose nanocrystals surface-modified via covalent grafting or physisorption: A comparative study. ACS Appl Mater Inter 9 (40): 35305-35318.
13. Aziz T, Fan H, Khan F, Ullah R, Haq F, et al. (2019) Synthesis of carboxymethyl starch-bio-based epoxy resin and their impact on mechanical properties. Zeitschrift für Physikalische Chemie 234(11-12) 1759-1769.

14. Gardebjer S, Bergstrand A, Idstrom A, Borstell C, Naana S, et al. (2015) Solid-state NMR to quantify surface coverage and chain length of lactic acid modified cellulose nanocrystals, used as fillers in biodegradable composites. Compos Sci Technol 107: 1-9.

15. Jamil MI, Ali A, Haq F, Zhang Q, Zhan X, et al. (2018) Icephobic strategies and materials with superwettability: design principles and mechanism. Langmuir 34 (50): 15425-15444.

16. Hemraz U, A Campbell K, S Burdick J, Ckless K, Boluk Y, et al. (2015) Cationic poly(2-aminoethylmethacrylate) and poly( $\mathrm{N}$ -(2-aminoethylmethacrylamide) modified cellulose nanocrystals: synthesis, characterization, and cytotoxicity 16(1): 319-325.

17. Aziz T, Fan H, Zhang X, Khan FU, Fahad S, et al. (2020) Adhesive properties of bio-based epoxy resin reinforced by cellulose nanocrystal additives. J Polym Eng 40 (4): 314-320.

18. Cao J, Fan H, Li BG, Zhu SP (2017) Synthesis and evaluation of doubledecker silsesquioxanes as modifying agent for epoxy resin. Polymer 124: 157-167.

19. Aziz T, Fan H, Haq F, Khan FU, Numan A, et al. (2019) Facile modification and application of cellulose nanocrystals. Iranian Polymer Journal 28 (8): 707-724.

20. Parameswaranpillai J, Ramanan SP, George JJ, Jose S, Zachariah AK, et al. (2018) PEG-ran-PPG modified epoxy thermosets: A simple approach to develop tough shape memory polymers. Industrial \& Engineering Chemistry Research 57 (10): 3583-3590.

21. Aziz T, Fan H, Zhang X, Khan FU (2019) Synergistic impact of cellulose nanocrystals and calcium sulfate fillers on adhesion behavior of epoxy resin. Mater Res Express 6 (11): 1150b1157.

22. Ricciardi MR, Papa I, Langella A, Langella T, Lopresto V, et al. (2018) Mechanical properties of glass fibre composites based on nitrile rubber toughened modified epoxy resin. Composites Part B-Engineering 139: 259-267.

23. Aziz T, Fan H, Zhang X, Haq F, Ullah A, et al. (2020) Advance study of cellulose nanocrystals properties and applications. J Polym Environ 28 (4): $1117-1128$.

24. Tan JQ Liu WQ, Wang ZF (2017) Hydrophobic epoxy resins modified by low concentrations of comb-shaped fluorinated reactive modifier. Progress in Organic Coatings 105: 353-361.

25. Yahyaei H, Ebrahimi M, Tahami HV, Mafi ER, Akbarinezhad E, et al. (2017) Toughening mechanisms of rubber modified thin film epoxy resins: Part 2-study of abrasion, thermal and corrosion resistance. Progress in Organic Coatings 113: 136-142.

26. Li C, Fan H, Aziz T, Bittencourt C, Wu L, et al. (2018) Biobased epoxy resin with low electrical permissivity and flame retardancy: from environmental friendly high-throughput synthesis to properties. ACS Sustain Chem Eng 6 (7): 8856-8867.

27. Zhou J, Jiang YY, Wu GQ, Wu WJ, Wang Y, et al. (2017) Investigation of dielectric and thermal conductive properties of epoxy resins modified by core-shell structured PS@SiO2. Composites Part a-Applied Science and Manufacturing 97: 76-82.

28. Aziz T, Iqbal M, Jamil MI, Ullah A, Ullah R, et al. (2020) Synthesis of isatin and its derivatives and their applications in biological system. Biomedical Journal of Scientific \&amp; Technical Research 30 (4): 23615-23621.

29. Abdollahi A, Roghani-Mamagani H, Salami-Kalajahi M, Mousavi A, Razavi B, et al. (2018) Preparation of organic-inorganic hybrid nanocomposites from chemically modified epoxy and novolac resins and silicaattached carbon nanotubes by sol-gel process: investigation of thermal degradation and stability. Progress in Organic Coatings 117: 154-165. 
30. Chen G, Feng JH, Qiu W, Zhao YM (2017) Eugenol-modified polysiloxanes as effective anticorrosion additives for epoxy resin coatings. RCS Advances 7 (88): 55967-55976.

31. Aziz T, Fan H, Ullah R, Haq F, Khan FU, et al. (2020) Facile synthesis and synergistic effect of calcium sulfate fillers on the adhesive properties of bisphenol A (DGEBA) epoxy resin. 2:3. 0342-0350.

32. Lu QL, Li XY, Tang LR, Lu BL, Huang B, et al. (2015) One-pot tandem reactions for the preparation of esterified cellulose nanocrystals with 4-dimethylaminopyridine as a catalyst. RSC Adv 5 (69): 56198-56204.

33. Aziz T, Fan H, Zhang XW, Khan FU (2019) Synergistic impact of cellulose nanocrystals and calcium sulfate fillers on adhesion behavior of epoxy resin. Mater Res Express 6 (11): 1-12.

34. Du LX, Wang JW, Zhang Y, Qi CS, Wolcott MP, et al. (2017) Preparation and characterization of cellulose nanocrystals from the bio-ethanol residuals. Nanomaterials 7 (3). 1-13.

35. Al-Turaif HA (2013) Relationship between tensile properties and film formation kinetics of epoxy resin reinforced with nanofibrillated cellulose. Progress in Organic Coatings 76 (2-3): 477-481.

36. Ferreira JAM, Reis PNB, Costa JDM, Capela C (2014) Assessment of the mechanical properties of nanoclays enhanced low Tg epoxy resins. Fibers and Polymers 15 (8): 1677-1684.

ISSN: 2574-1241

DOI: 10.26717/BJSTR.2021.34.005492

Tariq Aziz. Biomed J Sci \& Tech Res

(CC) This work is licensed under Creative

Submission Link: https://biomedres.us/submit-manuscript.php
37. Pinheiro IF, Ferreira FV, Souza DHS, Gouveia RF, Lona LMF, et al. (2017) Mechanical, rheological and degradation properties of PBAT nanocomposites reinforced by functionalized cellulose nanocrystals. Eur Polym J 97: 356-365.

38. McManus JB, Yang H, Wilson L, Kubicki JD, Tien M, et al. (2018) Initiation, elongation, and termination of bacterial cellulose synthesis. ACS Omega 3 (3): 2690-2698.

39. Madani A, Kiiskinen H, Olson JA, Martinez DM (2011) Fractionation of microfibrillated cellulose and its effects on tensile index and elongation of paper. Nord Pulp Pap Res J 26 (3): 306-311.

40. Bai LM, Bossa N, Qu FS, Winglee J, Li GB, et al. (2017) Comparison of hydrophilicity and mechanical properties of nanocomposite membranes with cellulose nanocrystals and carbon nanotubes. Environ Sci Technol 51 (1): 253-262.

41. Mustapha R, Rahmat AR, Majid RA, Mustapha SNH (2018) Mechanical and thermal properties of montmorrillonite nanoclay reinforced epoxy resin with bio-based hardener. Mater Today-Proc 5 (10): 21964-21972.

42. Que F, Khadr A, Wang GL, Li T, Wang YH, et al. (2018) Exogenous brassinosteroids altered cell length, gibberellin content, and cellulose deposition in promoting carrot petiole elongation. Plant Sci 277: 110120 .

$\begin{array}{ll}\text { BIOMEDICAL } & \text { Assets of Publishing with us } \\ \text { RESEARCHES } & \text { - Global archiving of articles } \\ & \text { - Immediate, unrestricted online access } \\ & \text { - Rigorous Peer Review Process } \\ \end{array}$

